\title{
A Large Cervical Immature Cystic Teratoma in a New-Born-Simulating Lymphatic Malformation
}

\begin{abstract}
Cervical teratomas are rare and challenging tumors. Although most are benign in nature, there location near vital neck structures can cause significant morbidity. Sometimes, making a diagnosis can be a challenge, especially when it is purely cystic and closely resembles lymphatic malformation.
\end{abstract}

Keywords: Cervical teratoma, cystic neck mass, new-born

\section{Introduction}

Cervical teratomas are rare tumors comprising only $3 \%-5 \%$ of all pediatric teratomas, with the incidence of 1 in 20,000-1 in 40,000 live births. ${ }^{[1,2]}$ They are usually diagnosed on antenatal scan as neck mass or postnatally as cervical mass with or without airway compromise. Although mostly benign in nature, large lesions can cause perinatal mortality because of upper airway obstruction and so require an extensive perinatal planning for upper airway management.

The diagnosis of teratoma can be challenging, especially if it is a purely cystic teratoma as then it becomes difficult to differentiate it from lymphatic malformation.

We present a rare case of cervical cystic immature teratoma in a newborn resembling lymphangioma.

\section{Case Report}

This was a 1-day-old, $2.7 \mathrm{~kg}$ full-term female child, born with cesarean delivery. The child was referred to us with complaints of large midline cervical mass with stridor. The antenatal history was uneventful. The child had mild respiratory distress and was accepting feeds well. On examination, there was a large neck mass of size about $10 \mathrm{~cm} \times 12 \mathrm{~cm}$, firm in consistency, and noncompressible involving almost whole of the neck [Figure 1].

This is an open access journal, and articles are distributed under the terms of the Creative Common Attribution-NonCommercial-ShareAlike 4.0 License, which allows others to remix, tweak, and build upon the work non-commercially, as long as appropriate credit is given and the new creations are licensed under the identical terms.

For reprints contact: WKHLRPMedknow_reprints@wolterskluwer.com
The clinical findings were suggestive of tumor. However, on ultrasound and MR angiogram [Figure 2], it was a well-defined lesion with multiloculated cystic areas without calcification. There was some internal hemorrhage, and it was a non-enhancing lesion on the arterial and venous phase. The mass was indenting the trachea. A very likely possibility of lymphatic malformation was given. Serum alphafetoprotein (AFP) levels were $35,000 \mathrm{ng} / \mathrm{ml}$.

With a high suspicion of cystic teratoma in view of a firm, well defined, noncompressible, and noninfiltrative lesion, the baby was planned for the surgery. Intraoperatively, it was large thick-walled lobulated mass involving bilateral submandibular triangles and submental triangle [Figure 3]. It was adherent densely posteriorly with the thyroid gland and pretracheal fascia. The mass was completely excised. The postoperative period was uneventful. The child was shifted on ventilator and was extubated after $48 \mathrm{~h}$.

On gross examination, the specimen was of size $10 \mathrm{~cm} \times 8 \mathrm{~cm}$ with multiple cystic areas and mucoid contents. Microscopic examination showed neuroglial tissue, cartilage, squamoid epithelium, focal calcification, and small foci of primitive neuroepithelial tissue suggestive of immature teratoma Grade II.

After follow-up of 1 year, child is fine with no recurrence and normal alpha feto protein and normal thyroid profile.

How to cite this article: Jain P, Prasad A, Prasad A Jain S. A large cervical immature cystic teratoma in a new-born-simulating lymphatic malformation. Indian J Med Paediatr Oncol 2020;41:615-7.

\section{Prashant Jain ${ }^{1}$, Ashish Prasad', Aparna Prasad², Sarika Jain ${ }^{3}$}

${ }^{1}$ Department of Pediatric Surgery, BLK Superspeciality Hospital, New Delhi, India, ${ }^{2}$ Department of Neonatology, BLK Superspeciality Hospital, New Delhi, India, ${ }^{3}$ Department of Radiodiagnosis, DODA Imaging, New Delhi, India

Submitted: 01-Apr-2020 Revised: $30-\mathrm{Apr}-2020$

Accepted: 01-Jun-2020 Published: 29-Aug-2020

Address for correspondence: Dr. Prashant Jain, 39/12, East Patel Nagar, New Delhi - 110 008, India. E-mail: docpedsurg@gmail.com

Access this article online Website: www.ijmpo.org DOI: 10.4103/ijmpo.ijmpo_120_20 Quick Response Code:

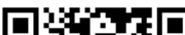

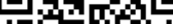
+7+1't Giver

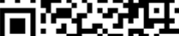




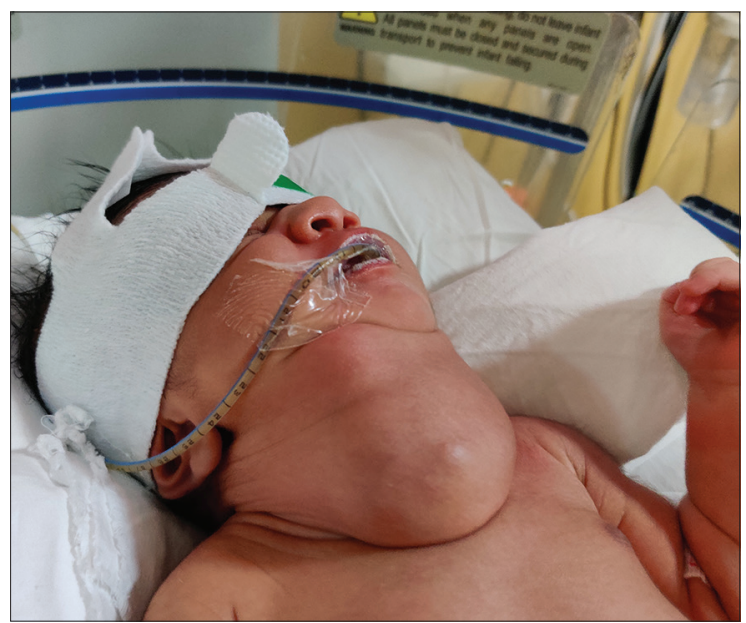

Figure 1: Newborn with a large cystic teratoma

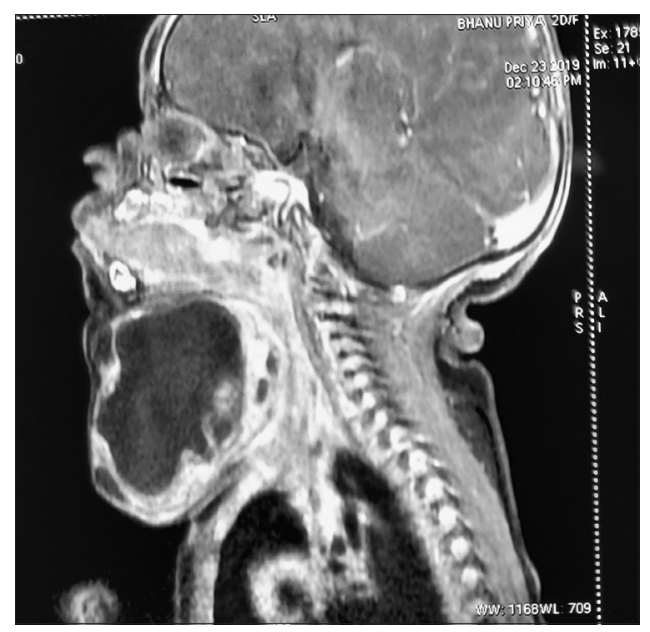

Figure 2: Magnetic resonance imaging suggestive of multiloculated cystic mass without any solid component and calcification

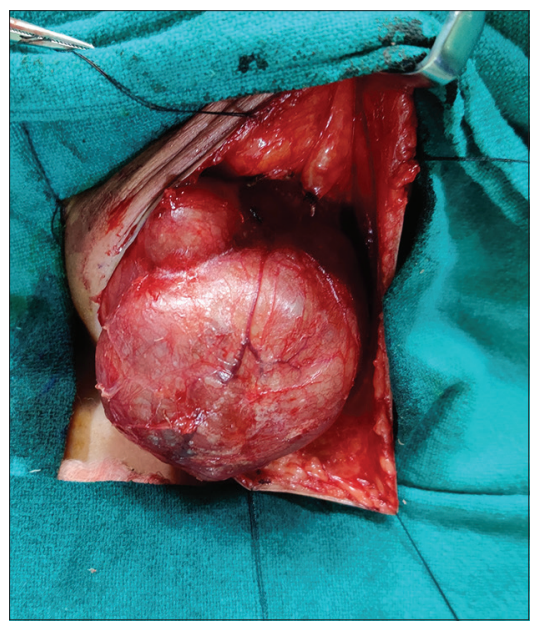

Figure 3: Intraoperative picture showing large lobulated mass adherent posteriorly to thyroid tissue and pretracheal facia

\section{Discussion}

Congenital cervical teratomas are rare in newborns, accounting for $1 \%-5 \%$ of all pediatric teratomas. ${ }^{[1,2]}$ They predominate in females. ${ }^{[2]}$ Large cervical teratomas may extend into the mediastinum or compress trachea, causing pulmonary hypoplasia and many of these may require an extensive perinatal multidisciplinary planning for EXIT (exutero intrapartum treatment) procedure. If left untreated, cervical teratomas have an estimated mortality rate of $80 \%-100 \%{ }^{[3]}$

The diagnosis of the cystic teratoma can be confusing, especially if it is predominantly cystic lesion as then it may resemble lymphatic malformation. Both are located in the anterior and posterior triangle of the neck which further add to the diagnostic challenge. The risk of airway compromise with teratomas is more as they are more firm and less compressible than lymphangioma. When the diagnosis is in question, a high-resolution magnetic resonance imaging (MRI) or computed tomography scan can help in confirming the diagnosis; however, in some cases, it can be challenging. MRI is preferred modality as it is radiation free and provides better soft tissue detail. ${ }^{[4,5]}$

On ultrasound, cervical lymphangiomas appear as infiltrating cystic lesion with fine internal septations. While cervical teratomas are usually thick walled, well-defined noninfiltrating lesions with solid and cystic components. The characteristics of thick wall with solid and cystic components and the presence of calcification, which is seen in $50 \%$ of cases, are useful in distinguishing a cervical teratoma from lymphangioma. ${ }^{[5]}$ The unique imaging characteristics are helpful in making the diagnosis but sometimes can be confusing as was in our case.

Some studies have suggested that origin of neck teratomas is from embryonic cells in primitive thyroid gland which explains there close relationship with thyroid tissue requiring thyroidectomy in some cases. ${ }^{[4,6]}$ After surgical removal, cervical teratoma patients should be evaluated for hypothyroidism. In a series of 23 children presented to Great Ormond Street Hospital, London, with a head and neck teratoma, 5 cases of neck teratoma were found to have origin from the thyroid gland. ${ }^{[7]}$ In our case, also the origin of mass was from the thyroid tissue.

Serum AFP level is a tumor marker for making the diagnosis, but its interpretation can be fallacious as it can normally be elevated in the neonatal period and can vary depending upon the age and birth weight. ${ }^{[4,7]}$

Prompt surgical excision of a teratoma is the treatment of choice. If needed, stabilization of airway should be done before the surgery. Cervical teratomas in children are almost always benign and rarely can be immature or malignant. However, it has been reported that immaturity of various constituent tissues in childhood teratomas (unlike adult) does not impact on the otherwise favorable prognosis of the teratoma. ${ }^{[8]}$ Chemotherapy is indicated in case of recurrence, disseminated disease, invasive tumor, incomplete resection, and malignancy. ${ }^{[8,9]}$ The disease and 
recurrence can be monitored by serial AFP measurements at regular intervals..$^{[3]}$

Few prognostic factors have been reviewed by Alexender et $a .^{[7]}$ which includes polyhydramnios and the tracheoesophageal index (sum of the lateral and ventral displacements of the tracheoesophageal complex from its normal anatomical location at the ventral aspect of the cervical spine) which have been found to be associated with the increased risk of neonatal respiratory compromise.

Long-term outcome in these patients has been found to be excellent after complete surgical excision. ${ }^{[4]}$ Outcome is certainly excellent when compared to lymphatic malformations in terms of quality of life, persistent or recurrent disease. ${ }^{[10]}$

\section{Conclusion}

A large neck mass in the neonatal period requires a high index of suspicion based on the clinical features and imaging to differentiate a teratoma from a lymphangioma as prompt surgical excision is mandatory in the former.

Although usually benign, cervical teratomas carry a significant risk of intrauterine or neonatal death because of polyhydramnios and upper airway obstruction. A good planning and imaging ensures complete excision without any significant morbidity.

\section{Declaration of patient consent}

The authors certify that they have obtained all appropriate patient consent forms. In the form, the legal guardian has given his consent for images and other clinical information to be reported in the journal. The guardian understands that names and initials will not be published and due efforts will be made to conceal identity, but anonymity cannot be guaranteed.
Financial support and sponsorship

Nil.

\section{Conflicts of interest}

There are no conflicts of interest.

\section{References}

1. Bianchi B, Ferri A, Silini EM, Magnani C, Sesenna E. Congenital cervical teratoma: A case report. J Oral Maxillofac Surg 2010;68:667-70.

2. Tonni G, De Felice C, Centini G, Ginanneschi C. Cervical and oral teratoma in the fetus: A systematic review of etiology, pathology, diagnosis, treatment and prognosis. Arch Gynecol Obstetrics 2010;282:355-61.

3. Paradis J, Koltai PJ. Pediatric teratoma and dermoid cysts. Otolaryngol Clin North Am 2015;48:121-36.

4. Neff L, Cervical teratomas. J Operative Techniques Otolaryngol 2017;28:190-5.

5. Gezer HÖ, Oğuzkurt P, Temiz A, Bolat FA, Hiçsönmez A. Huge neck masses causing respiratory distress in neonates: Two cases of congenital cervical teratoma. Pediatr Neonatol 2016;57:526-30.

6. Shetty KJ, Kishan Prasad HL, Rai S, Kumar YS, Bhat S, Sajjan N, et al. Unusual presentation of immature teratoma of the neck: A rare case report. J Cancer Res Ther 2015;11:647.

7. Alexander VR, Manjaly JG, Pepper CM, Ifeacho SN, Hewitt RJ, Hartley BE. Head and neck teratomas in children-a series of 23 cases at Great Ormond Street Hospital. Int J Pediatric Otorhinolaryngol 2015;79:2008-14.

8. Chakravarti A, Shashidhar TB, Naglot S, Sahni JK. Head and neck teratomas in children: A case series. Indian J Otolaryngol Head Neck Surg 2011;63:193-7.

9. Azizkhan RG, Haase GM, Applebaum H, Dillon PW, Coran AG, King PA, et al. Diagnosis, management, and outcome of cervicofacial teratomas in neonates: A Childrens Cancer Group study. J Pediatr Surg 1995;30:312-6.

10. Sheikh F, Akinkuotu A, Olutoye OO, Pimpalwar S, Cassady CI, Fernandes CJ, et al. Prenatally diagnosed neck masses: Long-term outcomes and quality of life. J Pediatric Surg 2015;50:1210-13. 